\title{
Impact of hormone receptor, HER2, and Ki-67 status conversions on survival after neoadjuvant chemotherapy in breast cancer patients: a retrospective study
}

\author{
Yuhai Chen ${ }^{1 \#}$, Xiaoyan Liu ${ }^{2 \#}$, Keda Yu ${ }^{3}$, Xiangyu Sun ${ }^{1}$, Shouping Xu', Pengfei Qiu ${ }^{5}$ Zhidong $\mathrm{Lv}^{6}$, \\ Xinwen Zhang ${ }^{7}$, Ayao Guo ${ }^{1}$, Yingying $\mathrm{Xu}^{1}$
}

${ }^{1}$ Department of Breast Surgery, The First Affiliated Hospital of China Medical University, Shenyang, China; ${ }^{2}$ Department of Breast Surgery, Cancer Hospital of China Medical University, Liaoning Cancer Hospital \& Institute, Shenyang, China; ${ }^{3}$ Department of Breast Surgery, Shanghai Cancer Center and Cancer Institute, Shanghai Medical College, Fudan University, Shanghai, China; ${ }^{4}$ Department of Breast Surgery, Harbin Medical University Cancer Hospital, Harbin, China; ${ }^{5}$ Breast Cancer Center, Shandong Cancer Hospital and Institute, Shandong First Medical University and Shandong Academy of Medical Science, Jinan, China; ${ }^{6}$ Breast Center, The Affiliated Hospital of Qingdao University, Qingdao, China; ${ }^{7}$ Center of Implant Dentistry, School and Hospital of Stomatology, China Medical University, Liaoning Provincial Key Laboratory of Oral Disease, Shenyang, China

Contributions: (I) Conception and design: Y Chen, X Liu, Y Xu, A Guo; (II) Administrative support: Y Xu, A Guo, K Yu, X Sun, S Xu, P Qiu, Z Lv, X Zhang; (III) Provision of study materials or patients: Y Chen, X Liu; (IV) Collection and assembly of data: Y Chen, X Liu; (V) Data analysis and interpretation: Y Chen, X Liu; (VI) Manuscript writing: All authors; (VII) Final approval of manuscript: All authors.

"These authors contributed equally to this work and should be considered as co-first authors.

Correspondence to: Yingying Xu; Ayao Guo. Department of Breast Surgery, The First Affiliated Hospital of China Medical University, Shenyang 110001, China. Email: xuyingying@cmu.edu.cn; ayguo@cmu.edu.cn.

Background: The discordance of estrogen receptor (ER), progesterone receptor (PR), human epidermal growth factor receptor 2 (HER2), and Ki-67 cell nuclear proliferation antigen status in patients with locally advanced breast cancer pre- and post-neoadjuvant chemotherapy (NAC) is quite common. This study aimed to assess the frequency of changes in receptor status after NAC in patients with invasive ductal breast cancer and the prognostic impact of such changes.

Methods: The study comprised 670 patients who were diagnosed with invasive ductal breast carcinoma and treated with both NAC and surgery from 2012-2017. Hormone receptor (HR; including ER and PR), HER2, and Ki-67 status was assessed before NAC and in residual invasive tumor cells of the surgical specimens. The prognostic impact of receptor conversions in breast cancer patients treated with NAC was evaluated in this retrospective study.

Results: The conversion of ER was related to overall survival (OS; $\mathrm{P}=0.008$ ) and disease-free survival (DFS; $\mathrm{P}=0.004)$. Patients whose ER status was always positive had the best prognosis, and those who were always negative had the worst prognosis. Similar results were also found for PR status, as the conversion of PR status was also related to OS $(\mathrm{P}<0.001)$ and DFS $(\mathrm{P}<0.001)$. At the same time, the conversion of Ki-67 status was related to OS $(\mathrm{P}=0.042)$ and $\mathrm{DFS}(\mathrm{P}=0.037)$, and patients whose $\mathrm{Ki}-67$ status was $\leq 20 \%$ persistently after NAC had the best survival among the 4 groups, while those whose Ki- 67 status changed from $\leq 20 \%$ to $>20 \%$ after NAC had the worst survival. Nevertheless, there was no statistical significance in the conversion of HER2 status. In multivariate Cox regression analyses, PR conversion and post-neoadjuvant pathological lymph node stage $(\mathrm{ypN})$ were independent prognostic factors for DFS $(\mathrm{P}=0.008,<0.001)$ and OS $(\mathrm{P}=0.002$, $<0.001)$.

Conclusions: Changes in receptor status between pre-treatment and residual disease after NAC are common. Moreover, these alterations have an impact on the survival outcome of invasive ductal breast cancer patients. Thus, receptor status should be re-evaluated routinely before and after NAC to guide individualized treatment. 
Keywords: Neoadjuvant chemotherapy (NAC); breast cancer; receptor conversions; prognostic impact

Submitted Dec 01, 2021. Accepted for publication Jan 14, 2022.

doi: $10.21037 /$ atm-21-6924

View this article at: https://dx.doi.org/10.21037/atm-21-6924

\section{Introduction}

Neoadjuvant chemotherapy (NAC) has been regarded as a standard treatment for breast cancer before surgery, and is beneficial for tumor downstaging, increasing the rate of breast-conserving surgery, and assessing the response of the tumor to chemotherapy $(1,2)$. Accumulating evidence has indicated that pathological complete response (pCR) following NAC serves as a crucial surrogate marker for prolonged survival outcomes and is therefore widely used as the primary endpoint in multiple NAC trials (3-5). In fact, most patients have residual disease at surgery, while only a small group of patients reach a pCR after NAC (5).

The prognosis and therapeutic response of breast cancer patients are significantly correlated with the expression status of the estrogen receptor (ER), progesterone receptor (PR), human epidermal growth factor receptor 2 (HER2), and $\mathrm{Ki}-67$. The role of HR is to formulate the regimen of endocrine therapy, and for HER2, is that whether it is suitable to carry out targeted therapy. Both of the receptors, with the addition of $\mathrm{Ki}-67$, can divide the breast cancer (BC) into several subtypes. Therefore, we can draw up a regimen of chemotherapy for each of the patients accordingly. All of the therapy can prevent tumor recurrence and metastasis in order to increase the rate of overall survival (OS) and disease-free survival (DFS) (6). Additionally, the receptor status before the initiation of NAC is required for the subsequent treatment planning. Therefore, in order to devise accurate individual NAC treatments according to the characteristics of tumors, routine examinations are performed to evaluate the status of ER, PR, HER2, and Ki-67 of the primary tumors. Meanwhile, the status of these biomarkers after NAC is also important for determining adjuvant systemic therapy. For instance, if patients had increased expression of hormone receptor (HR), HER2, and Ki-67 following NAC, and examinations for the immunohistochemical status in surgical specimens had not been performed, it may lead to a poor clinical outcome due to the lack of adjuvant hormonal treatment, anti-HER2 regimens, and adjuvant chemotherapy after surgery.

In this research, we have discovered discordance in the status of HR, HER2 and Ki-67 in patients with locally advanced breast cancer pre- and post-NAC, which means the status of HR and HER2 changed from positive to negative, negative to positive or even be stable, for Ki-67, the status changed from $\leq 20 \%$ to $>20 \%,>20 \%$ to $\leq 20 \%$, persistent $\leq 20 \%$ or persistent $>20 \%$. In previous studies, accumulating evidence has indicated a discordance in the expression of ER, PR, HER2, and Ki-67 pre- and post-NAC, and this conversion may serve as a potential prognostic factor, but the researchers just described a phenomenon about how the biomarkers change, and they didn't illustrate why the change happened (7-10). Determining the relationship between the discordance in receptor expression status pre- and after NAC and longterm survival outcomes prompts us to not only evaluate the response of the breast tumor to NAC but to adjust appropriate adjuvant treatments for breast cancer patients, but the previous researches simply divide the receptor status of study population into 2 group: stable and converted, which is incomplete to evaluate the relationship between conversion of receptor and OS, DFS (11). In this study, we innovatively divided the status of each receptor into four group and evaluated the relationship between discordance in status of receptors pre- and after NAC and long-term survival outcomes of patients with breast cancer. And we found out that PR conversion and ypN were independent prognostic factors for DFS and OS. Additionally, the frequency of receptor conversion after NAC varies in different studies. The discrepancy of receptor status between pre-surgery core needle biopsy (CNB) and surgical excision samples raises the question of whether retesting the receptor status post-NAC should be routinely performed. We present the following article in accordance with the STROBE reporting checklist (available at https:// atm.amegroups.com/article/view/10.21037/atm-21-6924/rc).

\section{Methods}

The objective of this study was to assess the discordance of ER, PR, HER2, and Ki-67 status between CNB and 
surgical excision specimens in patients with breast cancer and to determine their effects on long-term survival outcomes. The most significant aim was to determine whether these conversions may guide clinical practice. In the present study, we assessed pre-NAC and post-NAC biomarker status from 670 breast cancer patients. More importantly, the relationship between receptor conversion and long-term survival outcomes including disease-free survival (DFS) and overall survival (OS) was also evaluated in the present study.

\section{Study population}

We retrospectively collected data from patients diagnosed with primary breast cancer who received NAC and surgery at the Department of Breast Surgery, the First Affiliated Hospital of China Medical University and Cancer Hospital of China Medical University from February 2012 to September 2017. Data were stored in databases of both hospitals. We urged patients with breast cancer to undergo a review every 6 months. At the same time, with regard to patients who did not come to hospital on time, we organized a group to carry out follow-up by telephone. The median follow-up period was 52.0 months (ranging from 4.0 to 99.0 months).

\section{Treatment}

In this observational retrospective study, all patients received more than 1 cycle of NAC treatment using anthracycline-based regimens, taxane-based regimens, and anthracycline- and taxane-based regimens, followed by surgery with adjuvant radiotherapy, chemotherapy, and endocrine therapy whenever indicated. Twenty-five patients with HER2-positive breast cancer received trastuzumabcontaining NAC. Among 416 patients with HR-positive disease, $303(72.8 \%)$ received adjuvant endocrine therapy with tamoxifen or an aromatase inhibitor. All patients were registered in the hospital-based breast cancer registry and follow-up database system. All patients underwent a structured postoperative follow-up, with regular clinical visits and selected imaging studies yearly.

\section{Evaluation of $\mathrm{NAC}$ response and outcome}

We retrospectively identified demographic variables, pathological data from the biopsy before NAC, and the postoperative pathology report. Patient characteristics included age at diagnosis, menopausal status, body mass index (BMI), tumor size, post-neoadjuvant pathological lymph node stage (ypN), post-neoadjuvant pathological tumor stage (ypT), NAC regimens and cycles, clinical response to NAC, histological grade, and adjuvant therapies. The local and distant extent of disease was assessed by radiological examinations including breast ultrasound, mammography, breast magnetic resonance imaging (MRI), chest computed tomography (CT), bone scan, or abdominal ultrasound. The clinical response evaluation was based on the Response Evaluation Criteria in Solid Tumors (RECIST) v1.1. Partial response was defined as the sum of the longest diameter of the target lesion and the short axis of the target lymph node reduced by more than $30 \%$ compared with baseline. Progressive disease was defined as the sum of the longest diameter of the target tumor increasing at least $20 \%$, taking as the reference the smallest sum recorded and absolute increase of at least $5 \mathrm{~mm}$, or appearance of one or more new lesions, or unequivocal progression of non-target lesions. Stable disease was defined as neither progressive disease nor partial response criteria being met. We implemented immunohistochemistry on CNB and surgical excision specimens in patients with breast cancer, in order to obtain the frequency of changes of ER, PR, HER2 and Ki-67 pre- and after NAC.

\section{Immunobistochemistry}

The pathological assessments of morphological and biological features were performed in clinical laboratories of the First Affiliated Hospital of China Medical University and Cancer Hospital of China Medical University. Immunohistochemical analysis was performed on formalinfixed, paraffin-embedded tissue sections using standard procedures for breast tumor specimens obtained from CNB and surgical resections. ER, PR, and HER2 status and the Ki-67 index were evaluated by 2 experienced pathologists from the Department of Pathology independently. Positive ER and PR status was set at $1 \%$ of tumor cells with positive nuclear staining. Tumors were defined as HER2 overexpression in cases of membrane staining of $3+$ or amplified fluorescence in situ hybridization (FISH), while HER2 negativity was defined in cases of 0 (no membrane staining) or 1+ (weak and incomplete membrane staining) scores or 2+ plus negative FISH. Ki-67 expression was divided into high and low expression groups with a cutoff 
of $20 \%$. A pCR was defined as no invasive or in-situ tumor both in the resected breast specimens and sampled lymph nodes (ypT0 ypN0).

\section{Statistical analysis}

All statistical analyses were performed using SPSS (IBM, version 23). DFS and OS were set as the endpoints of our study. DFS was calculated from the date of surgery to the date of first event (locoregional relapse, distant relapse, or death) and OS was calculated from the date of surgery to the date of death or last follow-up. The distribution of continuous variables between 2 groups was compared using one-way ANOVA, whereas the association between 2 categorical variables was investigated with chi-squared and Fisher's exact tests. The Kaplan-Meier method was used to calculate survival outcomes, and subgroups were compared by the log-rank test. Cox proportional hazards models were performed to evaluate the correlation between the alteration of receptor status and relapse or survival risk. The hazard ratio and $95 \%$ confidence intervals (CI) were calculated for each variable using the Cox univariate model. Few of date missed, and which was instead of the average.

\section{Ethical statement}

All procedures performed in this study involving human participants were in accordance with the Declaration of Helsinki (as revised in 2013). For the reason that The First Affiliated Hospital of China Medical University was the dominant of both units, the study was approved by the Institutional Review Board (IRB) of The First Affiliated Hospital of China Medical University [No. (2019)2019-722]. As for Cancer Hospital of China Medical University, the IRB of this hospital did not implement an ethical review on retrospective study with single tumor of breast cancer. Individual consent for this retrospective analysis was waived.

\section{Results}

\section{Patient characteristics}

Although 1,192 patients were initially identified for inclusion in the study, 46 patients with bilateral breast cancer, 8 patients with inflammatory breast cancer, 58 patients with progression and metastatic disease prior to surgery, and 154 patients with incomplete information were excluded. Of the 926 patients who received NAC treatment, 170 cases (18.4\%) achieved pCR and 86 patients had insufficient residual tumor cells to evaluate the receptor status, and these patients were also excluded. To avoid heterogeneity, a total of 670 invasive ductal breast cancer patients with pre-NAC and matched surgical specimens were finally included in this study (Figure 1). We included 670 patients who fulfilled the study criteria at the Department of Breast Surgery in the First Affiliated Hospital of China Medical University and Cancer Hospital of China Medical University. Patient characteristics are shown in Table 1. After NAC treatment, 304 (45.3\%) patients had a change in at least one receptor. ER, PR and HER2 status was changed in 151 (22.5\%), 171 (25.5\%), and $161(24.0 \%)$ patients after NAC respectively. There were $586(87.5 \%)$ patients' Ki-67 status changed among 670 patients with invasive ductal breast carcinoma. At a median follow-up of 52.0 months (ranging from 4.0 to 99.0 months), 77 (11.5\%) of 670 patients had died, and 91 (13.6\%) of 670 patients had experienced disease recurrence.

Univariate analysis (Tables 2,3) identified that the conversion of ER, PR, and $\mathrm{Ki}-67$ status and ypN were significantly associated with both DFS and OS, and NAC cycles were also correlated with OS. The multivariate Cox proportional hazard analyses only included variables that were statistically significant $(\mathrm{P}<0.05)$ in univariate analyses. In multivariate Cox regression analyses (Tables 2,3), PR conversion and ypN were statistically significant for both DFS and OS.

\section{Survival analysis for ER conversion}

It has been shown that ER-positive status appears to be associated with better survival, but it is not known how receptor conversions affect the prognosis of breast cancer patients. Kaplan-Meier curves showed significantly greater DFS for patients with persistent ER positivity before and after NAC than the other 3 groups of patients $(\mathrm{P}=0.004$, Figure $2 A$ ). A similar significant difference was also observed for OS $(\mathrm{P}=0.008$, Figure 2B). The 5-year DFS and 5-year OS estimates for patients with persistent ER positivity were $88 \%$ and $92 \%$, respectively. Patients with ER negative conversion $(78 \%$ and $81 \%)$, patients with ER positive conversion (85\% and $83 \%$ ), and patients with persistent ER negativity (78\% and $79 \%$ ) differed significantly.

\section{Survival analysis for $P R$ conversion}

With regard to $\mathrm{PR}$ conversion groups, patients who 


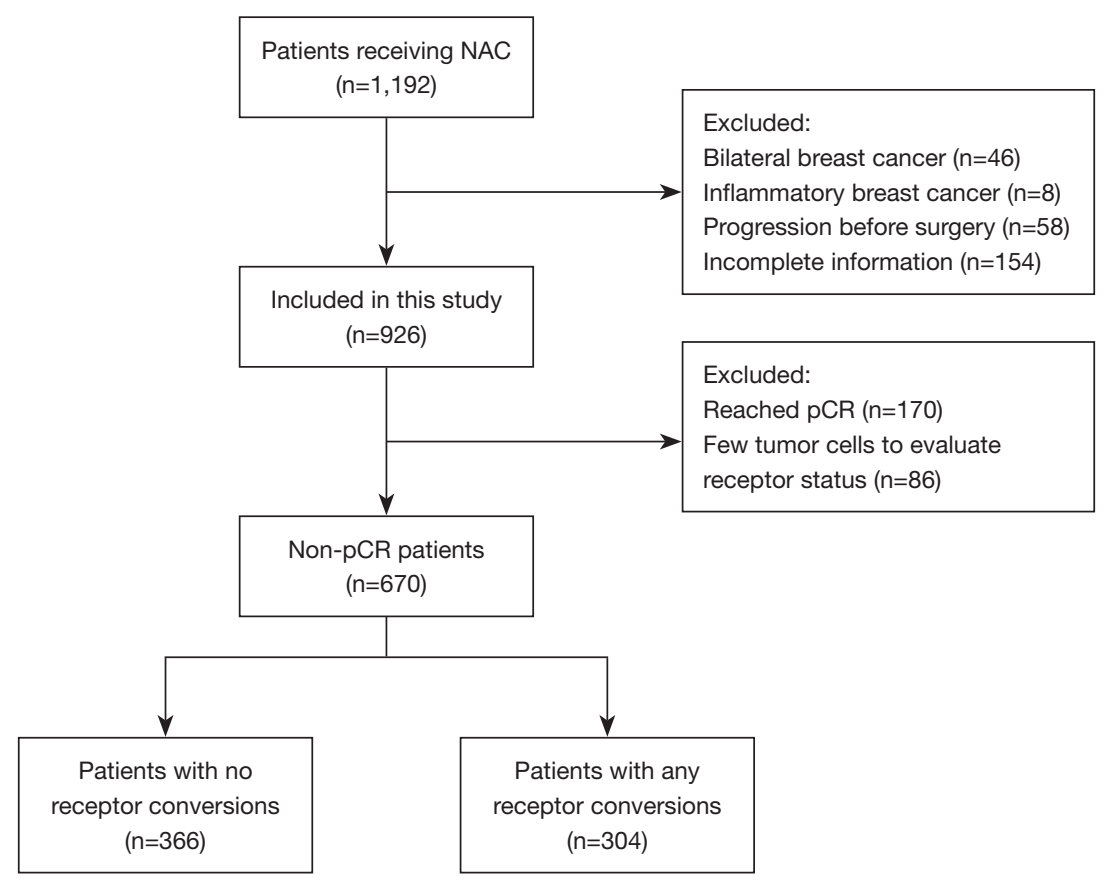

Figure 1 Flowchart showing the inclusion of patients in this study. NAC, neoadjuvant chemotherapy; pCR, pathologic complete response.

remained $\mathrm{PR}$ positive after NAC had better survival in terms of both DFS $(\mathrm{P}<0.001$, Figure $3 A)$ and $\mathrm{OS}(\mathrm{P}<0.001$, Figure $3 B)$. The 5 -year DFS and 5 -year OS estimates for patients with persistent $\mathrm{PR}$ positivity were $91 \%$ and $93 \%$, respectively. Patients with PR negative conversion $(85 \%$ and $87 \%$ ), patients with PR positive conversion ( $81 \%$ and $81 \%$ ), and patients with persistent PR negativity ( $78 \%$ and $80 \%$ ) differed significantly.

\section{Survival analysis for HER2 conversion}

According to HER2 status, no significant survival differences were found in the 4 HER2 conversion groups in terms of DFS $(\mathrm{P}=0.264$, Figure $4 A)$ and $\mathrm{OS}(\mathrm{P}=0.108$, Figure $4 B)$. The 5-year DFS and 5-year OS estimates for patients with persistent HER 2 positivity were $88 \%$ and $92 \%$, respectively. Patients with HER2 negative conversion (78\% and $80 \%)$, patients with HER2 positive conversion ( $82 \%$ and $85 \%$ ), and patients with persistent HER2 negativity $(84 \%$ and $86 \%)$ did not differ significantly.

\section{Survival analysis for Ki-67 conversion}

With regard to Ki-67 status, patients with persistent Ki-67 $\leq 20 \%$ before and after NAC had significantly greater DFS $(\mathrm{P}=0.042$, Figure $5 A)$ and $\mathrm{OS}(\mathrm{P}=0.037$, Figure 5B) compared with the other 3 groups of patients. The 5 -year DFS and 5-year OS estimates for patients with persistent Ki-67 $\leq 20 \%$ were $87 \%$ and $85 \%$, respectively. Patients with $\mathrm{Ki}-67$ increased conversion (68\% and 66\%), patients with Ki-67 decreased conversion (85\% and $87 \%$ ), and patients with persistent $\mathrm{Ki}-67>20 \%$ (69\% and $77 \%)$ differed significantly.

\section{Outcomes of immunobistochemistry}

The immunohistochemistry of $\mathrm{CNB}$ and surgical excision samples was conducted in clinical laboratories, we analysed and obtained the expression level of ER, PR, HER2 and Ki-67. In the clinical laboratories, ER positive status was set at $1 \%$ of tumor cells with positive nuclear staining (Figure S1A) and negative nuclear staining or less than $1 \%$ of tumor cells with positive nuclear staining represented ER negative (Figure S1B), which are in accordance with $\mathrm{PR}$ positive (Figure S1C) and negative (Figure S1D). The immunohistochemical staining of $\mathrm{Ki}-67$ can also be divided into 2 parts, is that $\leq 20 \%$ (Figure S1E) and $>20 \%$ (Figure S1F), in order to 
Table 1 Characteristics of patients with invasive ductal breast cancer

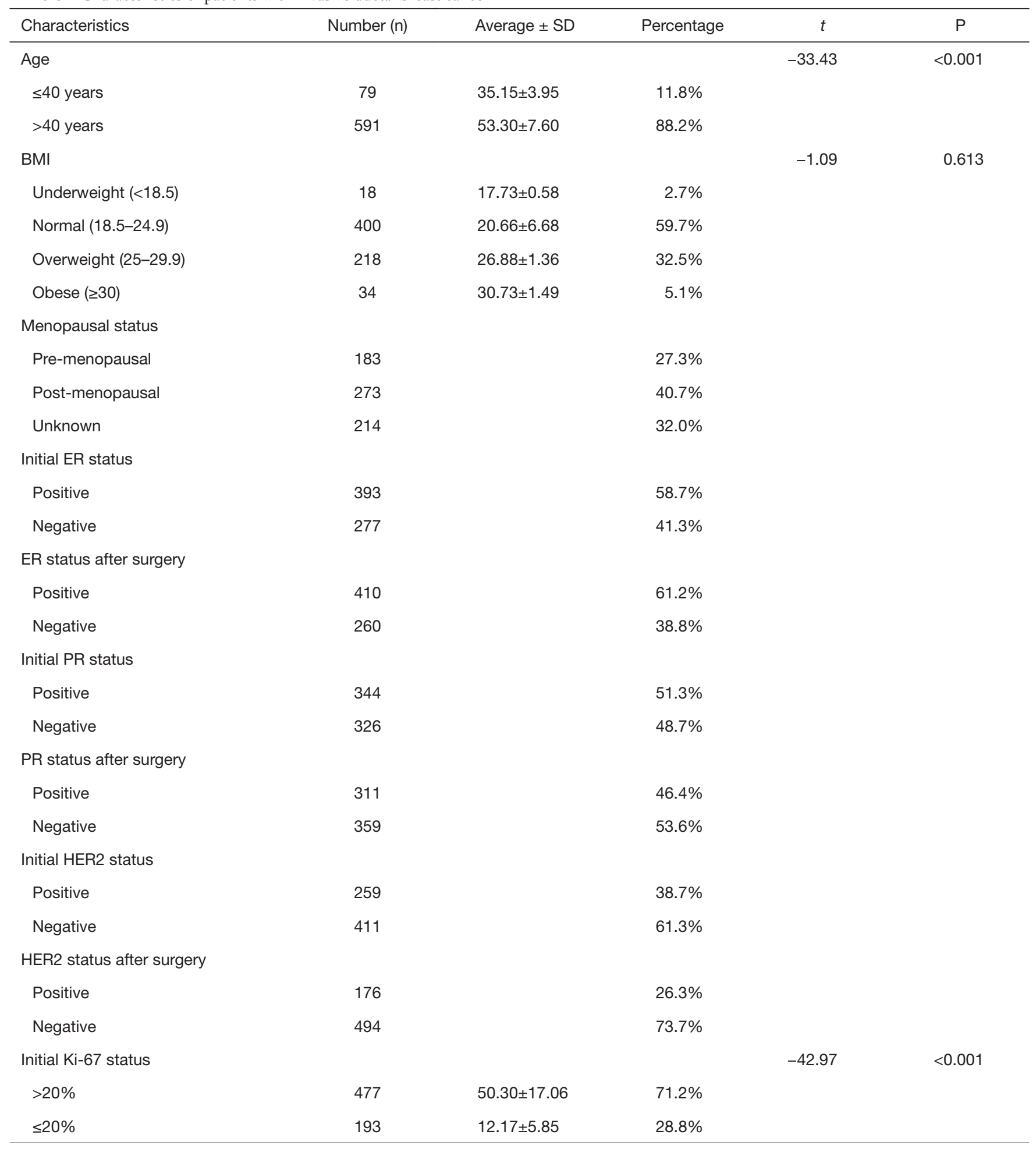

Table 1 (continued) 
Table 1 (continued)

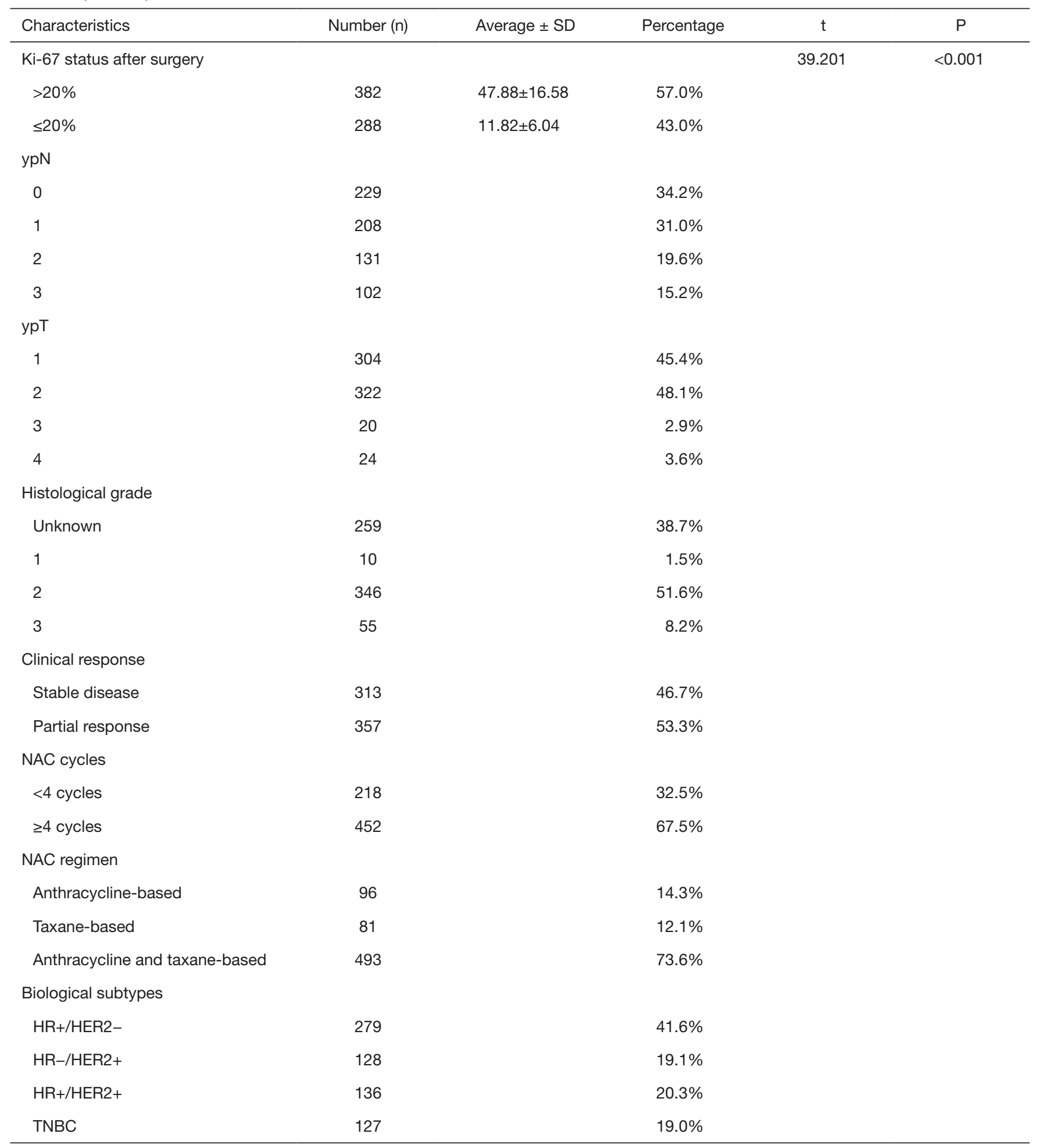

BMI, body mass index; ER, estrogen receptor; PR, progesterone receptor; HER2, human epidermal growth factor receptor-2; ypN, postneoadjuvant pathological node stage; ypT, post-neoadjuvant pathological tumor stage; NAC, neoadjuvant chemotherapy; HR, hormone receptor; TNBC, triple negative breast cancer. 
Table 2 Univariate and multivariate Cox regression analyses of disease-free survival

\begin{tabular}{|c|c|c|c|c|c|}
\hline Variables & Patients (n) & \multicolumn{2}{|c|}{ Univariate analysis } & \multicolumn{2}{|c|}{ Multivariate analysis } \\
\hline Age & & & 0.249 & & \\
\hline$\leq 40$ years & 79 & 1 & & & \\
\hline$>40$ years & 591 & $1.574(0.728-3.404)$ & & & \\
\hline Remained positive & 326 & 1 & & 1 & \\
\hline Remained negative & 193 & 2.285 (1.149-3.678) & 0.001 & $0.983(0.516-1.872)$ & 0.958 \\
\hline Positive to negative & 67 & $1.949(0.998-3.808)$ & 0.051 & $1.443(0.669-3.111)$ & 0.349 \\
\hline Negative to positive & 84 & $1.246(0.609-2.550)$ & 0.548 & $0.595(0.253-1.403)$ & 0.236 \\
\hline Remained negative & 257 & 3.525 (1.987-6.253) & $<0.001$ & 3.319 (1.587-6.939) & 0.001 \\
\hline Positive to negative & 102 & $1.909(0.893-4.078)$ & 0.095 & 1.607 (0.681-3.792) & 0.279 \\
\hline Negative to positive & 69 & $2.461(1.130-5.359)$ & 0.023 & 3.181 (1.265-7.999) & 0.014 \\
\hline HER2 conversion & & & 0.273 & & \\
\hline Remained positive & 137 & 1 & & & \\
\hline Remained negative & 372 & $1.370(0.743-2.525)$ & 0.313 & & \\
\hline Positive to negative & 122 & $1.938(0.981-3.826)$ & 0.057 & & \\
\hline Negative to positive & 39 & $1.523(0.578-4.010)$ & 0.395 & & \\
\hline$\leq 20 \%$ to $>20 \%$ & 78 & $0.896(0.519-1.893)$ & 0.128 & $1.256(0.899-2.354)$ & 0.523 \\
\hline ypN & & & $<0.001$ & & $<0.001$ \\
\hline 0 & 229 & 1 & & & \\
\hline 1 & 208 & $0.885(0.482-1.626)$ & 0.693 & $1.039(0.561-1.922)$ & 0.094 \\
\hline 2 & 131 & $1.659(0.924-2.979)$ & 0.090 & $1.860(1.029-3.363)$ & 0.040 \\
\hline 3 & 102 & $2.814(1.612-4.910)$ & $<0.001$ & $3.241(1.845-5.694)$ & $<0.001$ \\
\hline урт & & & 0.270 & & \\
\hline 1 & 304 & 1 & & & \\
\hline 2 & 322 & $1.269(0.817-1.969)$ & 0.289 & & \\
\hline 3 & 20 & $2.203(0.860-5.642)$ & 0.100 & & \\
\hline 4 & 24 & $1.805(0.703-4.632)$ & 0.220 & & \\
\hline
\end{tabular}

Table 2 (continued) 
Table 2 (continued)

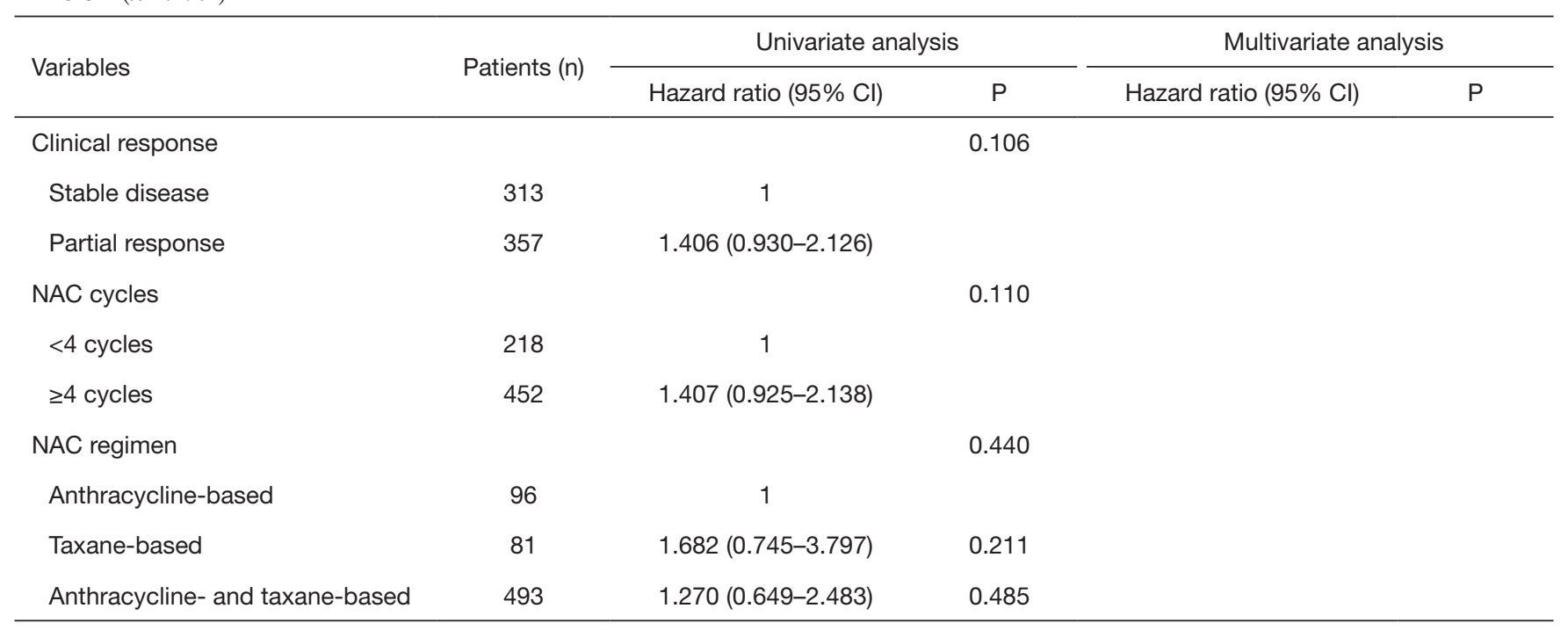

ER, estrogen receptor; PR, progesterone receptor; HER2, human epidermal growth factor receptor-2; ypN, post-neoadjuvant pathological node stage; ypT, post-neoadjuvant pathological tumor stage; NAC, neoadjuvant chemotherapy.

Table 3 Univariate and multivariate Cox regression analyses of overall survival

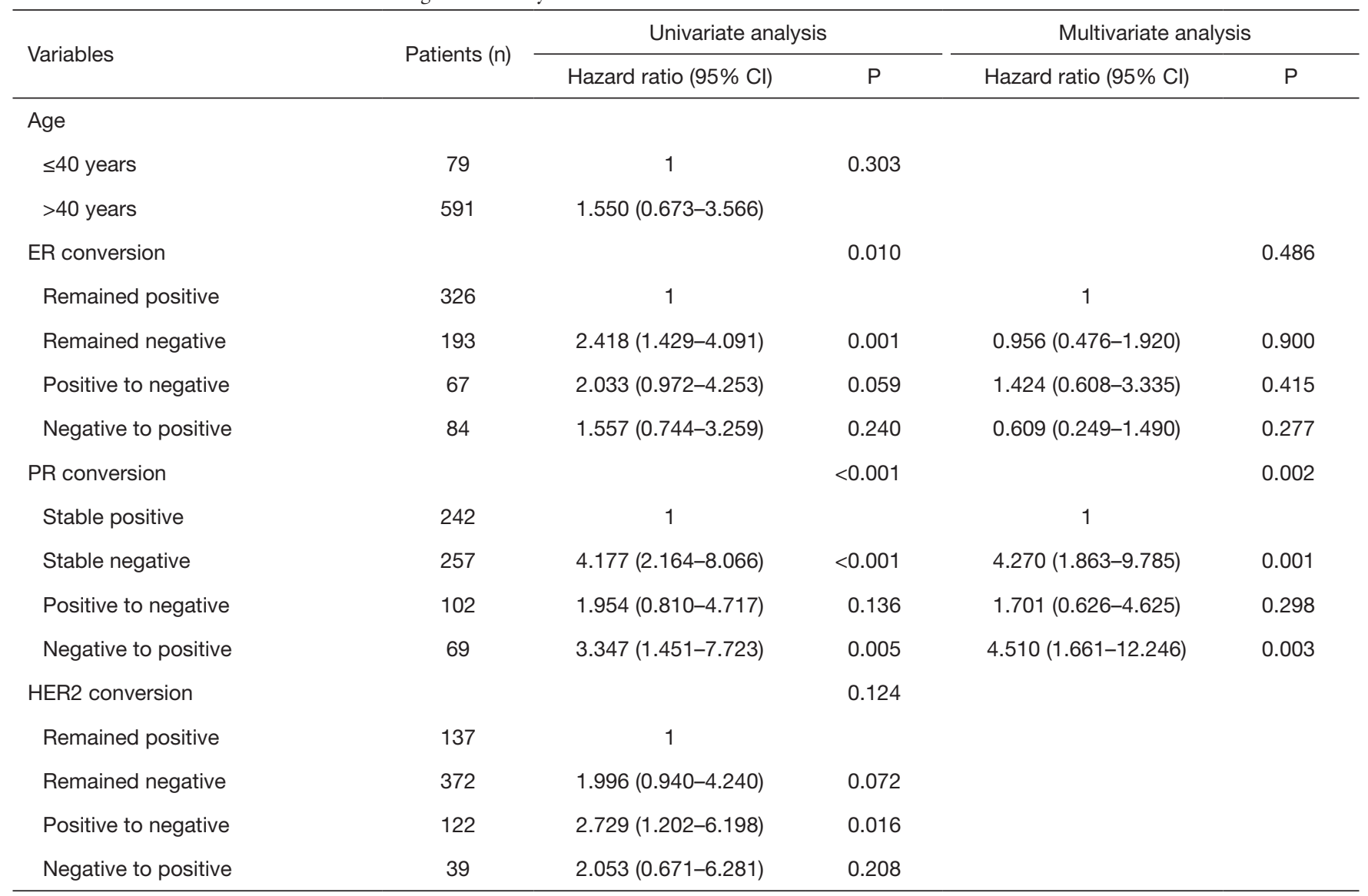

Table 3 (continued) 
Table 3 (continued)

\begin{tabular}{|c|c|c|c|c|c|}
\hline Variables & Patients (n) & \multicolumn{2}{|c|}{ Univariate analysis } & \multicolumn{2}{|c|}{ Multivariate analysis } \\
\hline $\mathrm{Ki}-67$ & & & 0.049 & & 0.300 \\
\hline Remained >20\% & 304 & 1 & & 1 & \\
\hline Remained $\leq 20 \%$ & 115 & $1.719(1.003-2.946)$ & 0.002 & $1.354(0.763-2.403)$ & 0.598 \\
\hline$\leq 20 \%$ to $>20 \%$ & 78 & $0.693(0.235-1.539)$ & 0.367 & $1.238(0.569-2.335)$ & 0.240 \\
\hline ypN & & & $<0.001$ & & $<0.001$ \\
\hline 0 & 229 & 1 & & 1 & \\
\hline 1 & 208 & $0.902(0.464-1.755)$ & 0.762 & $1.127(0.574-2.212)$ & 0.728 \\
\hline урт & & & 0.081 & & \\
\hline 1 & 304 & 1 & & & \\
\hline 2 & 322 & $1.428(0.876-2.327)$ & 0.153 & & \\
\hline 3 & 20 & 2.837 (1.088-7.395) & 0.033 & & \\
\hline 4 & 24 & $2.338(0.895-6.112)$ & 0.083 & & \\
\hline \multicolumn{6}{|l|}{ Clinical response } \\
\hline Stable disease & 313 & 1 & 0.102 & & \\
\hline Partial response & 357 & $1.456(0.928-2.284)$ & & & \\
\hline Taxane-based & 81 & $1.210(0.479-3.059)$ & 0.687 & & \\
\hline Anthracycline- and taxane-based & 493 & $1.250(0.615-2.542)$ & 0.537 & & \\
\hline
\end{tabular}

ER, estrogen receptor; PR, progesterone receptor; HER2, human epidermal growth factor receptor-2; ypN, post-neoadjuvant pathological node stage; ypT, post-neoadjuvant pathological tumor stage; NAC, neoadjuvant chemotherapy.

separate the molecular subtype of breast cancer patients. Tumors were defined as HER2 overexpression in cases of membrane staining of $3+$ or $2+$ plus amplified FISH (Figure S1G), while HER2 negativity was defined in cases of 0 (no membrane staining) or $1+$ (weak and incomplete membrane staining) scores or $2+$ plus negative FISH (Figure $\mathrm{S} 1 \mathrm{H}$ ).

\section{Discussion}

Currently, NAC is the standard therapeutic option for breast cancer. pCR has been identified as a surrogate endpoint for predicting long-term clinical benefit. pCR patients tend to have a favorable prognosis, and the residual tumors are reduced for re-testing the biomarker 
A

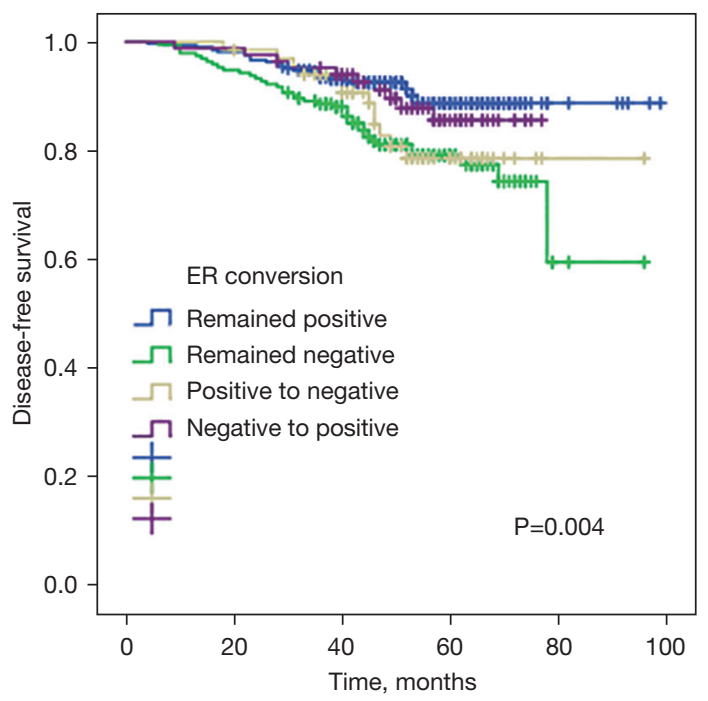

B

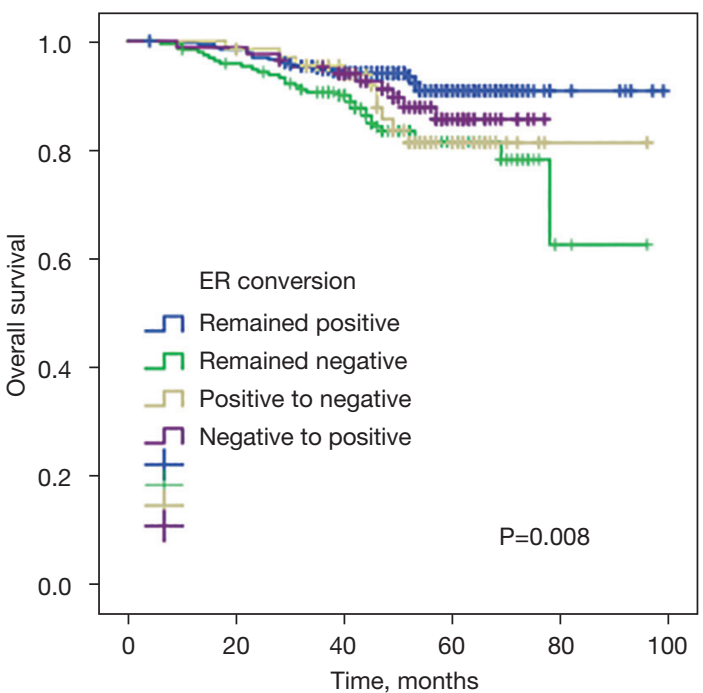

Figure 2 Disease-free survival (DFS) (A) and overall survival (OS) (B) curves according to estrogen receptor (ER) conversion.

A

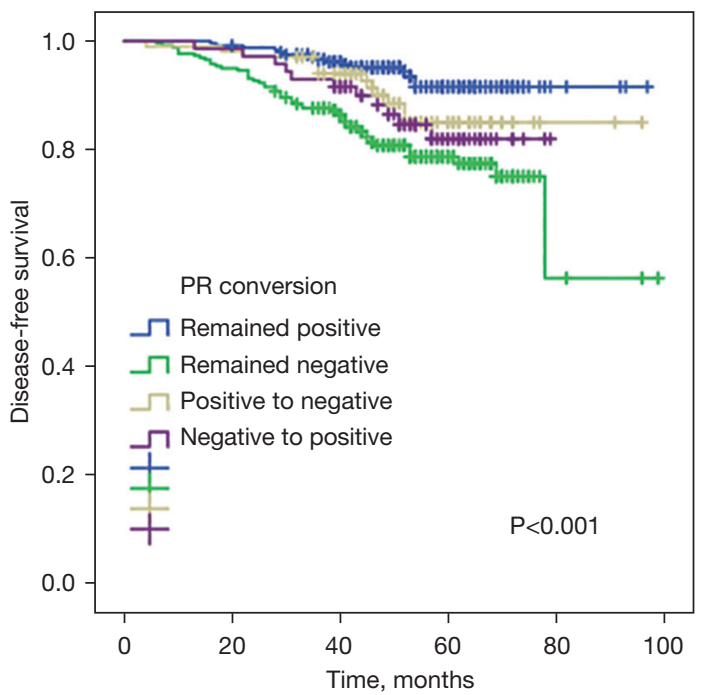

B

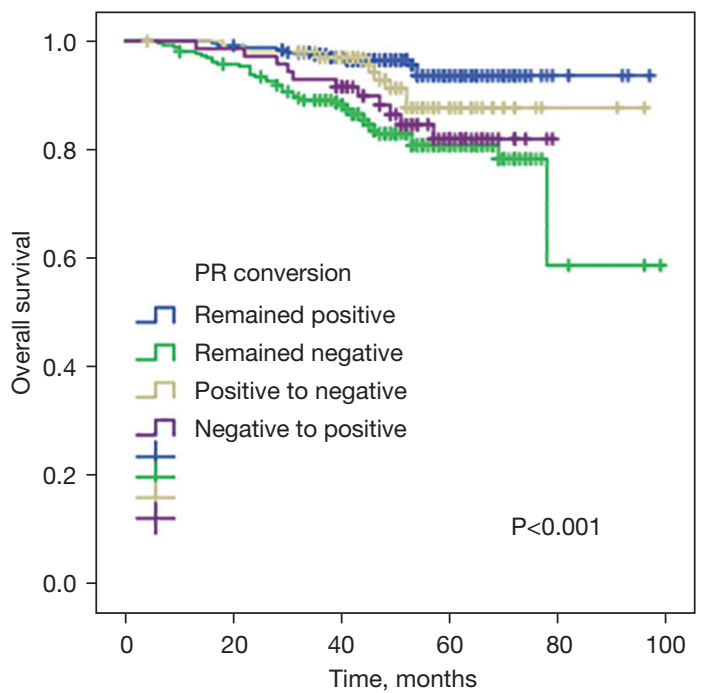

Figure 3 Disease-free survival (DFS) (A) and overall survival (OS) (B) curves according to progesterone receptor (PR) conversion.

expression post-NAC $(3,4)$. However, in non-pCR patients, the prognosis is worse, and it is crucial to find potential biomarkers to distinguish patients with different long-term outcomes. Numerous studies have reported discordances in ER, PR, HER2, and Ki-67 status pre- and post-NAC. In our study, we aimed to evaluate the discordance of receptor status in paired pre-surgery $\mathrm{CNB}$ and excision specimens in breast cancer patients treated with NAC, considering that receptor conversion may have crucial clinical implications for the prognosis of patients. Besides, receptor conversion may also have an impact on the selection of adjuvant chemotherapy, since altered receptor status after NAC may contribute to changing the subsequent adjuvant therapy regimen.

Changes in receptor and Ki-67 status occur for 2 distinct reasons. Firstly, the heterogeneity of the receptor 
A

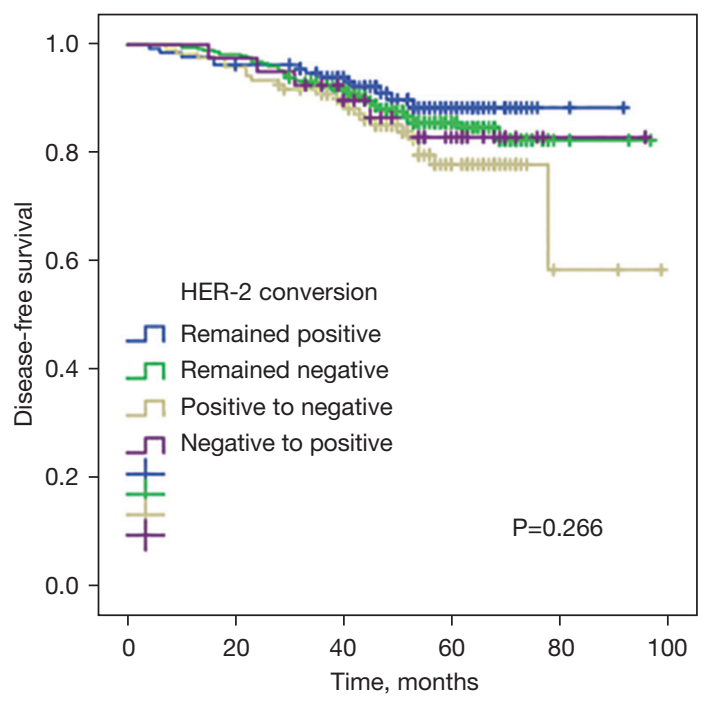

B

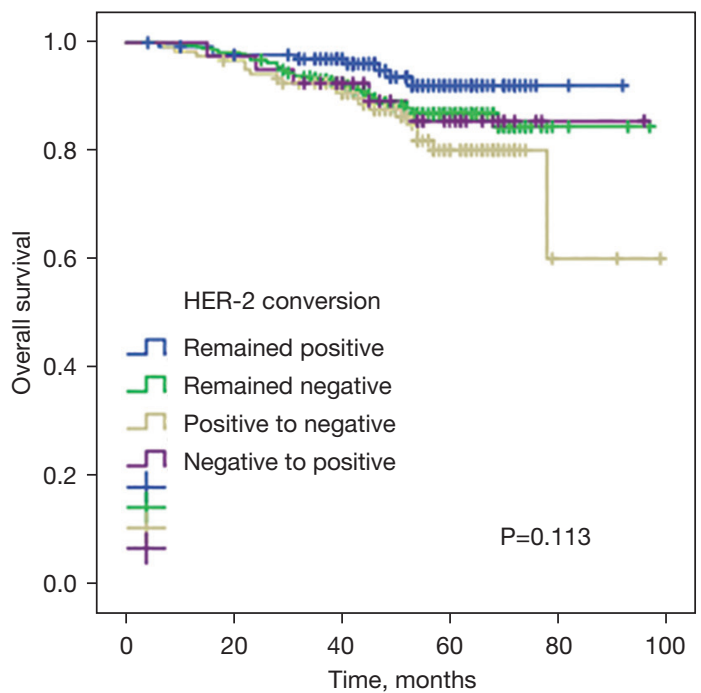

Figure 4 Disease-free survival (DFS) (A) and overall survival (OS) (B) curves according to human epidermal growth factor receptor-2 (HER2) conversion.

A

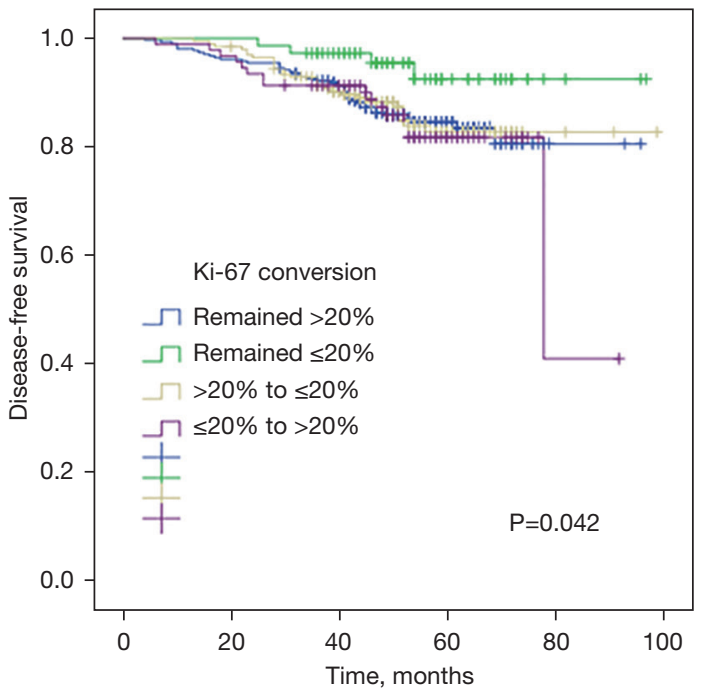

B

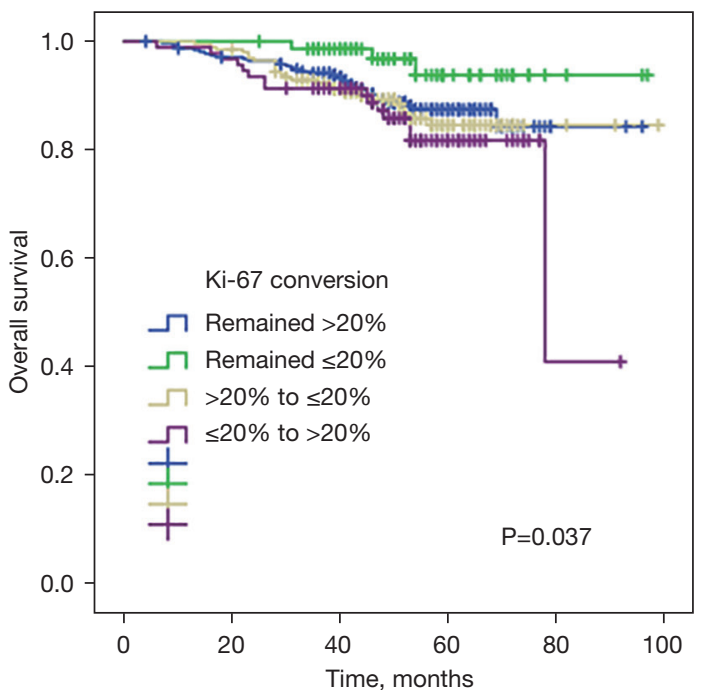

Figure 5 Disease-free survival (DFS) (A) and overall survival (OS) (B) curves according to Ki-67 conversion.

itself may result in the conversion of receptors and $\mathrm{Ki}-67$ status. The current understanding of the discordance in receptor status pre- and post-NAC is diverse and elusive. Undoubtedly, NAC therapy may induce alterations of gene expression profiles to some extent, and thus contribute to receptor conversion. For instance, negative conversion of
HR after NAC might be related to the decreasing level of hormones due to suppressed function of the ovaries (12). Given that sampling and detection techniques have significantly improved, intra-tumor heterogeneity may have a huge impact on receptor discordance $(13,14)$. Intratumor heterogeneity refers to distinct genetic, phenotypic, 
and behavioral features of cancer cells which coexist in the primary foci. For instance, different expression of ER, PR, HER2 and Ki-67 is observed not only in primary tumors between different regions, but also between primary tumors and other metastatic or recurrent lesions. Intra-tumoral heterogeneity is considered as a defining characteristic of breast tumors, thus the small tissue samples obtained by biopsy may not represent the characteristics of the entire tumor, and receptor discordance may result from sampling errors. Breast tumors with high heterogeneity were found to be correlated with less immune cell infiltration, weaker immune response, and worse survival outcomes (15). Additionally, ER heterogeneity serves as an independent long-term prognostic factor, which may have implications for therapeutic schedules, especially for luminal A subtype (16). However, because of tumor heterogeneity, we could not guarantee that every CNB sample or even surgical excision samples represented the overall receptor status (17). Secondly, there has been a shift in the test methods of molecular receptor expression levels-PAM50 to immunohistochemistry - which could also influence the result. In conclusion, there could be some small bias in the data, which warrants investigation as to how to avoid this.

The effect of HR status conversion on prognosis is still controversial. A meta-analysis showed that breast cancer patients with negative HR conversion displayed worse DFS and OS compared to patients with unchanged positive HR expression, which is consistent with our study (18). This reminds us that the loss of HR expression indicates greater aggressiveness of breast tumors. In fact, the conversion of HR status did not seem to be the only factor affecting the long-term survival outcome. The non-administration of adjuvant endocrine therapy may also correlate with a worse prognosis (19). Lim et al. found that although patients with positive HR expression were treated with adjuvant endocrine therapy, the survival outcomes of patients who converted from positive HR to negative HR were worse than those who remained HR positive (20). In a study which enrolled 231 patients with residual breast tumors after NAC, Yang et al. found that with adjuvant endocrine therapy after NAC and operation, patients with unchanged receptor status had significantly higher DFS than patients with any receptor conversion (21). Hirata $e t$ al. proposed that patients with changes in HR status after NAC but who did not receive endocrine therapy had worse survival outcomes than endocrine therapy-administered patients with unchanged positive HR (19). These results illustrate that it is necessary to evaluate the HR status of lesions not only in the pre-NAC CNB samples but also in surgical specimens after NAC, and hormonal therapy is suitable for patients with positive HR status conversion. Above all, whether conversion of HR status has crucial clinical implications for choice of adjuvant endocrine therapy is still unknown, especially when HR negative patients' receptor status turns into positive, so more attention should be paid to this topic.

The rates of HER2 discordance and its prognostic value has been widely investigated. Faneyte $e t$ al. proposed that HER2 status variation between CNB and surgical specimens was less frequent than HR alteration, suggesting that HER2 status may be more resistant to the modulatory effects of NAC (22). Besides, they also reported that positive conversion of HER2 status was more common than negative conversion. However, in the current study, we discovered that the rate of HER2 alteration was higher than ER conversion, and positive conversion was less common. These conflicting results may be explained by the different HER2 measurements and proportions of patients receiving anti-HER2 therapy. Multiple studies indicated that HER2 loss after NAC was correlated with a poorer prognosis $(8,23,24)$. Nevertheless, a large retrospective study comprising 588 non-pCR patients indicated that HER2 discordance has no effect on prognosis, regardless of HER2 status before NAC (25). These inconsistent results may be resolved by increasing the number of specimens in larger randomized clinical trials.

The effect of Ki-67 status conversion on prognosis is still controversial. $\mathrm{Li}$ et al. proposed that $\mathrm{Ki}-67$ before and after NAC, as well as the conversion, could predict the prognosis of breast cancer patients (17). However, it has yet to be determined how conversion affects the prognosis of patients with breast cancer. These results illustrate that it is necessary to evaluate the Ki-67 status of lesions not only in the pre-NAC CNB samples but also in surgical specimens after NAC, and chemotherapy is suitable for patients with Ki-67 status conversion ( $>20 \%$ to $\leq 20 \%$ ). Therefore, conversion of Ki-67 status may have crucial clinical implications for adjuvant chemotherapy especially when a patient with Ki-67 status $>20 \%$ changes to $\leq 20 \%$.

We acknowledge multiple limitations in the current study. Firstly, although the majority of patients were treated with standard chemotherapy, there were some difficulties in actual practice. Therefore, this may have introduced bias in the study, as a minority of patients did not receive the same 
NAC scheme. Secondly, various studies have demonstrated changed ER, PR, and HER2 status between CNB and surgical specimens without NAC treatment. Finally, this is a retrospective study, which should be categorized as realworld research, and some missing data exists in the current study due to the influence of the condition of patients. Given that the amount of missing data is small, the results may not be significantly influenced by this bias. Therefore, an additional trial that comprises a larger patient population treated with an identical therapeutic scheme should be conducted to further identify the prognostic value of receptor conversion after NAC in breast cancer.

For treatment to patients with breast cancer who may undergo a NAC, firstly, we should evaluate the status of receptors of CNB specimens, and implement NAC according to molecular subtype. Secondly, whether we should carry out the endocrine or targeted therapy and how to carry out based on the molecular subtype of surgical excision specimens. Therefore, not only the molecular subtype before NAC but after NAC does matter in the period of treatment, and the conversion in status of receptor can be a guidance to treatment options. For prognosis, the statistic outcomes differed in diverse molecular subtype. And the discordance in status of receptor pre- and after NAC portends diverse survival outcomes. Thus, receptor status should be re-evaluated routinely before and after NAC to guide individualized treatment.

\section{Conclusions}

The discordance rates of ER, PR, HER2, and Ki-67 status were $22.5 \%, 25.5 \%, 24.0 \%$, and $56.9 \%$ in patients after NAC, respectively. After NAC treatment, 304 $(45.3 \%)$ patients had a change in at least 1 receptor. We demonstrated that persistent ER positivity after NAC resulted in better survival in terms of both OS and DFS compared with the other 3 groups. Similar results were found for PR status. In contrast, Ki-67 status less than or equal to $20 \%$ had better survival in terms of both OS and DFS compared with the other 3 groups. In the multivariate Cox regression analysis, PR conversion was an independent prognostic factor for DFS and OS. Thus, receptor and Ki-67 status examination should be performed routinely after NAC. Understanding the alteration of receptors and Ki-67 status is not only of important prognostic value, it may also help make treatments for breast cancer more individualized.

\section{Acknowledgments}

Funding: This work was supported by the National Natural Science Foundation of China (No. 81773083), Chinese Young Breast Experts Research Project (No. CYBER2021-A02), Scientific and Technological Innovation Leading Talent Project of Liaoning Province (No. XLYC1802108), and Support Project for Young and Technological Innovation Talents of Shenyang (No. RC190393). These projects provided all the funds necessary for the collection of cases, the analyses of results, the statistical interpretation of the data, and the submission of the manuscript.

\section{Footnote}

Reporting Checklist: The authors have completed the STROBE reporting checklist. Available at https://atm. amegroups.com/article/view/10.21037/atm-21-6924/rc

Data Sharing Statement: Available at https://atm.amegroups. com/article/view/10.21037/atm-21-6924/dss

Conflicts of Interest: All authors have completed the ICMJE uniform disclosure form (available at https://atm. amegroups.com/article/view/10.21037/atm-21-6924/coif). The authors have no conflicts of interest to declare.

Ethical Statement: The authors are accountable for all aspects of the work in ensuring that questions related to the accuracy or integrity of any part of the work are appropriately investigated and resolved. All procedures performed in this study involving human participants were in accordance with the Declaration of Helsinki (as revised in 2013). For the reason that The First Affiliated Hospital of China Medical University was the dominant of both units, the study was approved by the Institutional Review Board (IRB) of The First Affiliated Hospital of China Medical University [No. (2019)2019-72-2]. As for Cancer Hospital of China Medical University, the IRB of this hospital did not implement an ethical review on retrospective study with single tumor of breast cancer. Individual consent for this retrospective analysis was waived.

Open Access Statement: This is an Open Access article distributed in accordance with the Creative Commons Attribution-NonCommercial-NoDerivs 4.0 International License (CC BY-NC-ND 4.0), which permits the non- 
commercial replication and distribution of the article with the strict proviso that no changes or edits are made and the original work is properly cited (including links to both the formal publication through the relevant DOI and the license). See: https://creativecommons.org/licenses/by-nc-nd/4.0/.

\section{References}

1. Karakatsanis A, Tasoulis MK, Wärnberg F, et al. Metaanalysis of neoadjuvant therapy and its impact in facilitating breast conservation in operable breast cancer. Br J Surg 2018;105:469-81.

2. Fisher B, Bryant J, Wolmark N, et al. Effect of preoperative chemotherapy on the outcome of women with operable breast cancer. J Clin Oncol 1998;16:2672-85.

3. Guarneri V, Broglio K, Kau SW, et al. Prognostic value of pathologic complete response after primary chemotherapy in relation to hormone receptor status and other factors. J Clin Oncol 2006;24:1037-44.

4. Cortazar P, Zhang L, Untch M, et al. Pathological complete response and long-term clinical benefit in breast cancer: the CTNeoBC pooled analysis. Lancet 2014;384:164-72.

5. von Minckwitz G, Untch M, Blohmer JU, et al. Definition and impact of pathologic complete response on prognosis after neoadjuvant chemotherapy in various intrinsic breast cancer subtypes. J Clin Oncol 2012;30:1796-804.

6. Prat A, Pineda E, Adamo B, et al. Clinical implications of the intrinsic molecular subtypes of breast cancer. Breast 2015;24 Suppl 2:S26-35.

7. Ahn S, Kim HJ, Kim M, et al. Negative Conversion of Progesterone Receptor Status after Primary Systemic Therapy Is Associated with Poor Clinical Outcome in Patients with Breast Cancer. Cancer Res Treat 2018;50:1418-32.

8. Parinyanitikul N, Lei X, Chavez-MacGregor M, et al. Receptor status change from primary to residual breast cancer after neoadjuvant chemotherapy and analysis of survival outcomes. Clin Breast Cancer 2015;15:153-60.

9. Ge WK, Yang B, Zuo WS, et al. Evaluation of hormone receptor, human epidermal growth factor receptor-2 and $\mathrm{Ki}-67$ with core needle biopsy and neoadjuvant chemotherapy effects in breast cancer patients. Thorac Cancer 2015;6:64-9.

10. van de Ven S, Smit VT, Dekker TJ, et al. Discordances in ER, PR and HER2 receptors after neoadjuvant chemotherapy in breast cancer. Cancer Treat Rev
2011;37:422-30.

11. Al-Saleh K, Salah T, Arafah M, et al. Prognostic significance of estrogen, progesterone and HER2 receptors' status conversion following neoadjuvant chemotherapy in patients with locally advanced breast cancer: Results from a tertiary Cancer Center in Saudi Arabia. PLoS One 2021;16:e0247802.

12. Bines J, Oleske DM, Cobleigh MA. Ovarian function in premenopausal women treated with adjuvant chemotherapy for breast cancer. J Clin Oncol 1996;14:1718-29.

13. Martelotto LG, Ng CK, Piscuoglio S, et al. Breast cancer intra-tumor heterogeneity. Breast Cancer Res 2014;16:210.

14. Yeo SK, Guan JL. Breast Cancer: Multiple Subtypes within a Tumor? Trends Cancer 2017;3:753-60.

15. McDonald KA, Kawaguchi T, Qi Q, et al. Tumor Heterogeneity Correlates with Less Immune Response and Worse Survival in Breast Cancer Patients. Ann Surg Oncol 2019;26:2191-9.

16. Lindström LS, Yau C, Czene K, et al. Intratumor Heterogeneity of the Estrogen Receptor and the Longterm Risk of Fatal Breast Cancer. J Natl Cancer Inst 2018;110:726-33.

17. Li L, Han D, Wang X, et al. Prognostic values of Ki-67 in neoadjuvant setting for breast cancer: a systematic review and meta-analysis. Future Oncol 2017;13:1021-34.

18. Li C, Fan H, Xiang Q, et al. Prognostic value of receptor status conversion following neoadjuvant chemotherapy in breast cancer patients: a systematic review and metaanalysis. Breast Cancer Res Treat 2019;178:497-504.

19. Hirata T, Shimizu C, Yonemori K, et al. Change in the hormone receptor status following administration of neoadjuvant chemotherapy and its impact on the longterm outcome in patients with primary breast cancer. Br J Cancer 2009;101:1529-36.

20. Lim SK, Lee MH, Park IH, et al. Impact of Molecular Subtype Conversion of Breast Cancers after Neoadjuvant Chemotherapy on Clinical Outcome. Cancer Res Treat 2016;48:133-41.

21. Yang L, Zhong X, Pu T, et al. Clinical significance and prognostic value of receptor conversion in hormone receptor positive breast cancers after neoadjuvant chemotherapy. World J Surg Oncol 2018;16:51.

22. Faneyte IF, Schrama JG, Peterse JL, et al. Breast cancer response to neoadjuvant chemotherapy: predictive markers and relation with outcome. Br J Cancer 2003;88:406-12.

23. Guarneri V, Dieci MV, Barbieri E, et al. Loss of HER2 
positivity and prognosis after neoadjuvant therapy in HER2-positive breast cancer patients. Ann Oncol 2013;24:2990-4.

24. Mittendorf EA, Wu Y, Scaltriti M, et al. Loss of HER2 amplification following trastuzumab-based neoadjuvant systemic therapy and survival outcomes. Clin Cancer Res 2009;15:7381-8.

Cite this article as: Chen Y, Liu X, Yu K, Sun X, Xu S, Qiu P, Lv Z, Zhang X, Guo A, Xu Y. Impact of hormone receptor, HER2, and $\mathrm{Ki}-67$ status conversions on survival after neoadjuvant chemotherapy in breast cancer patients: a retrospective study. Ann Transl Med 2022;10(2):93. doi: 10.21037/atm-21-6924
25. Yoshida A, Hayashi N, Suzuki K, et al. Change in HER2 status after neoadjuvant chemotherapy and the prognostic impact in patients with primary breast cancer. J Surg Oncol 2017;116:1021-8.

(English Language Editor: C. Betlazar-Mash) 


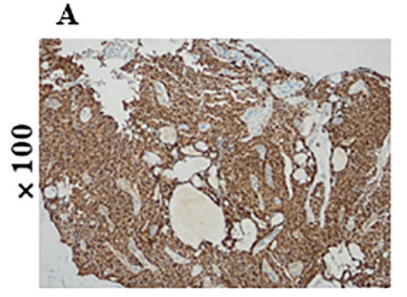

C

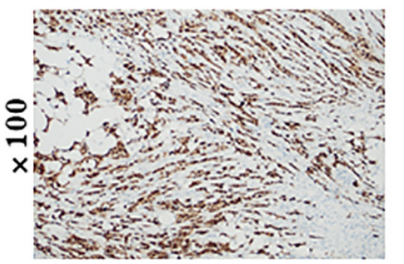

E

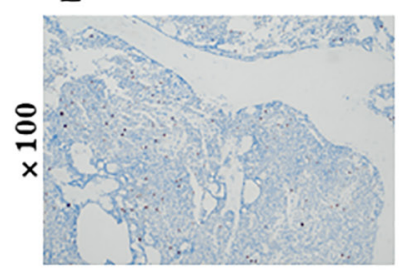

B

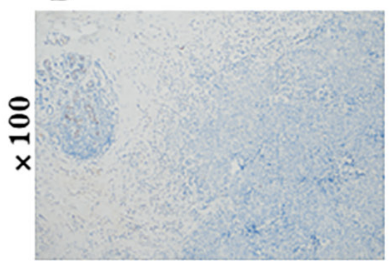

D
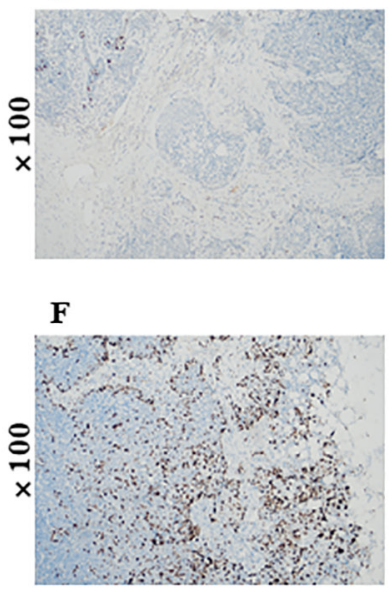

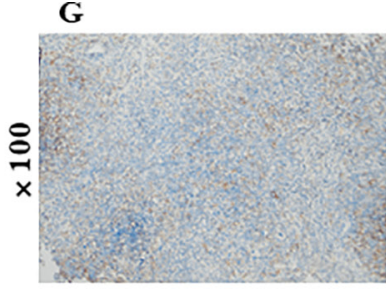

Score 2+

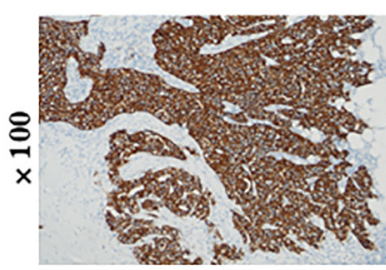

Score 3+

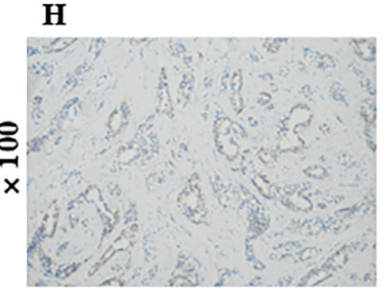

Score 2+

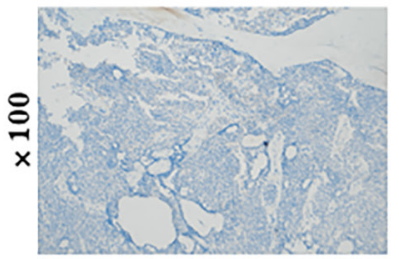

Score 0

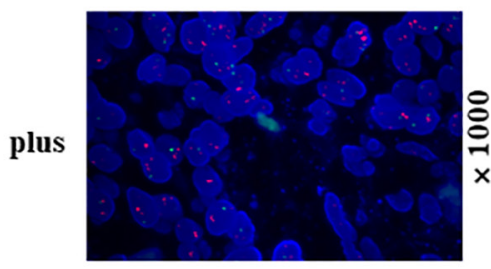

positive FISH

plus

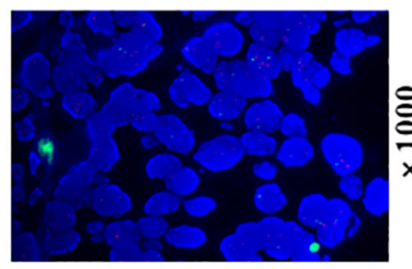

negative FISH

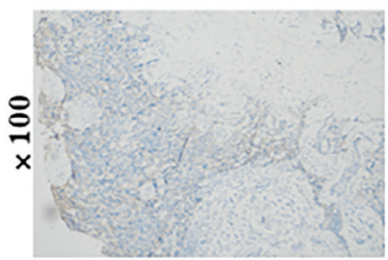

Score 1+

Figure S1 Representative immunohistochemical staining. ER positive (A) and ER negative (B), PR positive (C) and PR negative (D), Ki$67 \leq 20 \%$ (E) and Ki-67>20\% (F), HER2 positive (score 2+ plus positive FISH, score 3+, G) and HER2 negative (score 2+ plus negative FISH, score 0 and score 1+, H). ER, estrogen receptor; PR, progesterone receptor; HER2, human epidermal growth factor receptor-2; FISH, fluorescence in situ hybridization. 